\title{
Magnetic resonance imaging and clinical outcome in patients with symptomatic carotid artery stenosis after carotid artery revascularization
}

\author{
Rafał Badacz ${ }^{1}$, Anna Kabłak-Ziembicka ${ }^{1}$, Małgorzata Urbańczyk-Zawadzka², Robert P. Banyś'

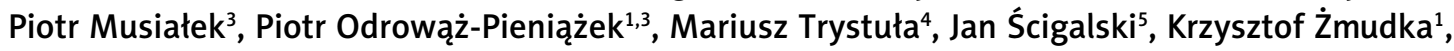 \\ Tadeusz Przewłocki ${ }^{1,3}$ \\ ${ }^{1}$ Department of Interventional Cardiology, Jagiellonian University Medical College, the John Paul II Hospital, Krakow, Poland \\ 2Department of Radiology and Diagnostic Imaging, the John Paul II Hospital, Krakow, Poland \\ ${ }^{3}$ Department of Cardiac and Vascular Diseases, Jagiellonian University Medical College, the John Paul II Hospital, Krakow, Poland \\ ${ }^{4}$ Department of Vascular and Endovascular Surgery, the John Paul II Hospital, Krakow, Poland \\ ${ }^{5}$ Department of Neurology and Stroke Unit, the Rydygier Hospital, Krakow, Poland
}

Adv Interv Cardiol 2017; 13, 3 (49): 225-232

DOI: https://doi.org/10.5114/aic.2017.70190

\begin{abstract}
A bstract
Introduction: About $30 \%$ of patients with carotid artery stenosis (CAS) develop dementia after a cerebral ischemic event (CIE), and $20-50 \%$ suffer from CIE recurrence during 6 months. Carotid artery revascularization (CAR) may prevent CIE recurrence, at the cost of new microembolic lesions (MES). The impact of CAR on cognitive function is debatable.

Aim: To assess functional and cognitive outcome, cerebral flow on transcranial Doppler (TCD) and brain magnetic resonance imaging (MRI) in patients with symptomatic CAS referred for CAR.

Material and methods: Twenty-two patients (aged $69.0 \pm 7.2$ y.o., 15 male) with recent CIE $(21.9 \pm 20.9$ days to CAR) related to CAS of mean $89.8 \pm 3.9 \%$ lumen reduction were prospectively evaluated with TCD, diffusion and perfusion MRI, Montreal Cognitive Assessment (MoCA), Mini Mental State Examination (MMSE), modified Rankin Scale (mRS) and the National Institutes of Health Stroke Scale (NIHSS) $24 \mathrm{~h}$ before, at 24-48 $\mathrm{h}$ and 1 month following CAR.

Results: New MES were found in 11 (50\%) subjects following CAR. CAR resulted in a significant increase of cerebral flow velocity in the middle and anterior cerebral arteries ( $p<0.002$ and $p=0.003$; respectively) and cerebral perfusion measured by time to peak (TTP) and mean transit time (MTT) ( $p=0.0009$ and $p=0.0002$; respectively). Neurologic tests showed improvement in NIHSS (2.4 \pm 1.6 to $1.5 \pm 1.2, p=0.003$ ), mRS (from $1.3 \pm 0.9$ to $0.7 \pm 0.9, p=0.005)$, and MMSE $(26.7 \pm 2.2$ to $27.6 \pm 2.3, p=0.019)$ at 1 month, while similar MoCA scores were observed before and 1 month after CAR $(23.4 \pm 3.3$ vs. $24.1 \pm 3.7, p=0.136)$.

Conclusions: Improvement of cerebral flow and perfusion and functional outcome, as well as at least no cognitive decline, is observed after CAR for symptomatic CAS.
\end{abstract}

Key words: symptomatic carotid artery stenosis, microembolic ischemic lesions, cerebral perfusion, magnetic resonance, transcranial color-coded Doppler, cognitive assessment.

\section{Introduction}

Carotid artery stenosis (CAS) plays an important role in cerebral ischemic event (CIE) incidence, e.g. ischemic stroke (IS) or transient ischemic accident (TIA), accounting for about $14 \%$ of all ISs according to the Oxford Vascular Study [1]. About one third of patients develop cognitive dementia following CIE [2], while $20-50 \%$ of subjects with symptomatic CAS suffer from CIE recurrence during
6 months [2-4]. Furthermore, CAS frequently co-exists with arterial occlusive disease in other vital territories, which also impacts the cardiovascular prognosis in this group of subjects $[5,6]$.

According to current guidelines prompt carotid artery revascularization (CAR) is recommended in patients with CAS exceeding $50 \%$ lumen reduction, preferably within 2 weeks following CIE $[7,8]$. This approach is associated with $33 \%$ recurrent $\mathrm{CIE}$ risk reduction for inter-

Corresponding author:

Anna Kabłak-Ziembicka MD, PhD, Department of Interventional Cardiology, Jagiellonian University Medical College, the John Paul II Hospital, 80 Prądnicka St, 31-202 Krakow, Poland, phone: +48 1261435 01, e-mail: kablakziembicka@op.pl

Received: 9.05.2017, accepted: 12.06.2017. 
ventional treatment as compared to the optimal medical approach [1].

Cerebral perfusion improvement seems a reasonable consequence of the restored cerebral flow after CAR, and this theoretically should be associated with an improvement in cognitive and functional status. Indeed, some researchers emphasize the role of cerebral perfusion improvement following CAR as a predictor of cognitive outcome [9].

However, the procedure either with carotid artery stenting (PTA) or carotid endarterectomy (CEA) may be potentially associated with further cognitive function decline, which may be attributed to new acute microembolic lesions (MES), noted on brain diffusion-weighted magnetic resonance imaging (DWI-MRI) or periprocedural transcranial color-coded duplex Doppler (TCD) monitoring [10-12]. On the other hand, some studies do not support the relationship between MES and cognitive function decline $[9,13]$. Moreover, according to some studies there is no association between periprocedural MES and cerebral perfusion [14-16].

\section{Aim}

This present study, covering a variety of aspects concerning the outcome of CAR either by CEA or by PTA, was aimed to assess functional and cognitive outcome, with regard to cerebral flow on TCD and DWI-MRI in patients with recently symptomatic CAS.

\section{Material and methods}

The study included 22 consecutive patients, (mean age: $69 \pm 7.2$ y.o., 15 male) with recent CIE $(21.9 \pm 20.9$ days to CAR, range: $5-89$ ) defined as first IS or TIA, related to severe CAS (mean stenosis degree: $89.8 \pm 3.9 \%$, range: $80-99 \%$ ), who were admitted to the Vascular and Endovascular Surgery Department between September 2015 and January 2017 with the aim of CAR.

The eligibility criteria were as follows: first CIE, unilateral, symptomatic CAS > 70\%, measured with carotid Duplex ultrasound, using the NASCET criteria, history of IS or TIA within 60 days prior to CAR.

The exclusion criteria were as follows: history of former CIE, patients with bilateral CAS, including contralateral carotid artery occlusion, known other potential causes of $\mathrm{CIE}$ (atrial fibrillation, patent foramen ovale, cerebral artery aneurysms/malformations), non-diagnostic temporal window for TCD, contraindications for MRI, known prior advanced dementia with Mini-Mental State Examination (MMSE) score below 20 and Montreal Cognitive Assessment Scale (MoCA) score below 14.

Each patient signed an informed consent form before the revascularization procedure. The study was performed according to the institutional bioethics committee and was performed in accordance with the Declaration of Helsinki.
At baseline, demographic data, presented symptoms, medical characteristics as well as cardiovascular risk factors (such as hypertension, hypercholesterolemia, diabetes mellitus, or active smoking) were collected from each patient. The obtained information included age, gender, history of coronary artery disease (CAD), myocardial infarction $(\mathrm{MI})$, history of coronary revascularization (both $\mathrm{PCl}$ and CABG), as well as interventions within peripheral arteries.

The qualifying IS or TIA was based on the consultant neurologist's opinion, and sourced from medical documentation and obtained brain imaging either with computed tomography (CT) or MRI.

\section{Internal carotid artery revascularization}

The patients admitted to the department were referred for PTA or CEA depending on the Vascular Team multidisciplinary decision, including the consultant neurologist. Carotid artery angiography was performed before CAR with the measurement of internal carotid artery lumen reduction using quantitative assessment (Siemens, Coroscop, Enlargen, Germany). The PTA was performed according to Tailored-CAS criteria, with a proximal or distal neuroprotection system in patients treated with dual antiplatelet therapy [17]. Stent and neuroprotection system selection was made according to degree of stenosis, plaque morphology and collateral cerebral flow [17]. The CEA was performed on a single antiplatelet agent and periprocedural heparin. The procedure technique choice was left at the discretion of the operating surgeon. All CEAs were performed with eversion technique and periprocedural shunt.

\section{Carotid ultrasonography}

The severity of CAS was assessed by high-resolution B-mode, color Doppler and pulse Doppler ultrasonography of extracranial arteries and was performed with an ultrasound machine (Toshiba Aplio TUS-A300; Saronno, Italy) fitted with a linear-array 7.5 MHz transducer. Patients were examined in a supine position with the head tilted backwards. In compliance with the Carpenter criteria [18], the grade of stenosis in carotid arteries was assessed by measuring the increase in the peak systolic velocity (PSV) $>2.1 \mathrm{~m} / \mathrm{s}$ and the end-diastolic velocity (EDV) $>0.7 \mathrm{~m} / \mathrm{s}$. Carotid plaque morphology was classified in accordance with Gray-Weale criteria [19], in brief: four plaque types were defined based on their degree of echolucency assessed in B-mode view: type 1: predominantly echolucent, type 2: intermediate echolucent lesions with small areas of echogenicity, type 3: intermediate echogenic lesions with small areas of echolucency, type 4: uniformly echogenic lesions. Furthermore, the plaque surface was described as either smooth, irregular or ulcerated.

\section{Transcranial color-coded duplex Doppler}

Following the carotid ultrasound, every patient had a TCD examination performed through the temporal 
approach. Examination of intracranial arteries was performed with a Toshiba Aplio TUS-A300; (Saronno, Italy) machine fitted with a sector-array $1.5-2.5 \mathrm{MHz}$ transducer. TCD examination included measurements of PSV and EDV in the first segments of cerebral arteries within the circle of Willis: ipsilateral to stenosed ICA in middle (MCA), and anterior (ACA) cerebral arteries, as well as contralateral MCA and ACA.

Furthermore, the flow directions and functions of anterior ( $\mathrm{ACOA}$ ) and posterior ( $\mathrm{PCOA})$ communicating arteries were assessed. The ACoA was assessed as present when the same flow directions in the ipsilateral MCA and ACA were observed. PCoA was assessed as present when the blood flow between the trifurcation and ipsilateral posterior cerebral artery (P1/P2 segment) was recorded on TCD [20]. All scans were obtained by the same experienced sonographer who had no prior knowledge of the subjects' clinical and angiographic characteristics.

Both carotid ultrasound and TCD were performed 1 day prior to CAR, at 24-48 h after CAR and 4 weeks following CAR.

\section{Magnetic resonance imaging}

To assess cerebral perfusion, magnetic resonance scans were obtained 1 day prior to CAR, at 24-48 $\mathrm{h}$ after CAR and 4 weeks following CAR.

The imaging was performed with a $1.5 \mathrm{~T}$ scanner (Magnetom Sonata Maestro Class, Erlangen, Germany), using a dedicated 8-element head coil. The protocol covered the DWI as well as perfusion-weighted imaging (PWI) with cerebral blood volume (CBV), cerebral blood flow (CBF), time to peak (TTP), and mean transit time (MTT), calculated upon acquired images. The scanning protocols included T1-weighted images, T2-weighted images and FLAIR images. Dynamic susceptibility contrast (DSC) MRI is also known as PWI.

On DWI imaging 24-48 $\mathrm{h}$ after CAR any new detectable lesions within both hemispheres, not visualized in the study prior to CAR, were considered as MES. The total number of lesions and maximal diameter were calculated manually by a qualified radiologist.

The PWI data were acquired using $\mathrm{T}^{*}$-weighted imaging by a gradient echo-planar sequence with the first passage of contrast bolus in brain tissues, after intravenous injection of a standard dose of $0.05 \mathrm{mmol} / \mathrm{kg}$ body weight of gadolinium-based contrast agent with the flow of $5 \mathrm{ml} / \mathrm{s}$ followed by a bolus of $20 \mathrm{ml}$ saline $(0.9 \% \mathrm{NaCl})$ with the same flow $(5 \mathrm{ml} / \mathrm{s})$ using an automatic power injector. During the first pass of gadolinium-based contrast agent it is confined in the vasculature and produces local magnetic field gradients that induce a decrease in the signal of T2*-weighted images (susceptibility effects). These susceptibility effects are seen as a transient signal loss in the tissue of interest on $\mathrm{T} 2^{*}$-weighted imaging.
Using commercially available software, various functional parameters and maps of TTP and MTT were calculated from the time-signal intensity curves measured in each pixel. Maps can be interpreted visually or quantitatively by calculating the relative values. The range of values of each perfusion parameter is demarcated by a color scale, usually with the color red indicating the highest values and blue representing the lowest values.

The regions of interest were set manually within the area of MCA parallel in both hemispheres as well as within the venous sinus. The assessment was made before CAR, 24-48 $\mathrm{h}$ after CAR and after 4 weeks of follow-up for the ipsilateral hemisphere. The mean values of MTT and TTP were calculated for each patient separately in each study.

\section{Cognitive function}

Cognitive function of the study participants was assessed with Montreal Cognitive Assessment (MoCA) [21, 22] and Mini Mental State Examination (MMSE) [23] scales, prior to CAR, 24-48 h after CAR and after 1-month of follow-up. The exclusion criteria were the cut-off score values equal to or below 20 and 14 for MMSE and MoCA, respectively, as suggesting severe dementia.

\section{Functional status}

Functional outcome was estimated using the modified Rankin Scale (mRS) and National Institutes of Health Stroke Scale (NIHSS) at $24 \mathrm{~h}$ before CAR, at 24-48 $\mathrm{h}$ after and at 1 month following CAR [24, 25].

\section{Statistical analysis}

Continuous variables are presented as mean \pm one SD, categorical variables are expressed as frequencies and percentages. Means of analyzed parameters across groups were tested with the analysis of variance (ANOVA) test, and frequencies were compared by the $\chi^{2}$ test for independence. The non-parametric Mann-Whitney $U$ test was used to determine differences in peak-systolic flow velocities recorded in the corresponding cerebral arteries among the studied group of patients as well as the differences in neuropsychological and functional tests' outcomes. The non-parametric Wilcoxon test for dependent samples was performed to assess the differences in PWI parameters: MTT and TTP in studies performed before and after CAR, the associations between PWI parameter changes and new MES following CAR, as well as the change in neuropsychological tests' outcomes before and after CAR. The correlation between brain PWI assessment (TTP and MTT values) on MRI and cerebral flow on TCD, as well as the correlation between appearance of new, acute MES following CAR and improvement of PWI parameters and neurocognitive test results, was calculated with the non-parametric Spearman's rank-order test. Statistical analyses were performed with Statis- 
tica 12.0 software. Statistical significance was assumed at $p<0.05$.

\section{Results}

Baseline characteristics of study participants are shown in Table I.

Upon assessment of carotid plaque morphology, the majority of plaques observed in study participants were considered high risk (type I and II), as evidenced by ultrasonography and post-CAR histological assessment of plaques (Table I).

The PTA was performed in 17 (77.3\%), CEA in 5 (22.7\%) subjects (Table II). Periprocedural 30-day complications occurred in 3 (13.6\%) subjects, including 2 (9.0\%) CIE (NIHSS 5, mRS 3 and NIHSS 2, mRS 1 respectively) after PTA observed within $24 \mathrm{~h}$ following CAR, the first one

Table I. Clinical characteristics of study participants

\begin{tabular}{|c|c|}
\hline Parameter & Value \\
\hline Number of patients & 22 \\
\hline Age & $69.0 \pm 7.2$, range: $56-81$ \\
\hline Male gender & $15(68.2 \%)$ \\
\hline Hypertension & $22(100 \%)$ \\
\hline Diabetes mellitus & $6(27.3 \%)$ \\
\hline Hyperlipidemia & $21(95.5 \%)$ \\
\hline Cigarette smoking - current or former & $7(31.8 \%)$ \\
\hline Coronary artery disease* & $13(59.1 \%)$ \\
\hline History of myocardial infarction & $1(4.5 \%)$ \\
\hline History of $\mathrm{PCl}$ & $3(13.6 \%)$ \\
\hline History of CABG & $1(4.5 \%)$ \\
\hline Peripheral arterial disease & $6(27.3 \%)$ \\
\hline Grade of ICAS before CAR ${ }^{\star *}$ & $\begin{array}{c}89.8 \pm 3.9 \% \\
\text { range: } 80-99 \%\end{array}$ \\
\hline String stenosis & $7(31.8 \%)$ \\
\hline \multicolumn{2}{|l|}{ Plaque morphology: } \\
\hline Type 1: Predominantly echolucent & $5(22.7 \%)$ \\
\hline Type 2: Intermediate echolucent & $10(45.5 \%)$ \\
\hline Type 3: Intermediate echogenic & $5(22.7 \%)$ \\
\hline Type 4: Uniformly echogenic & $2(9.1 \%)$ \\
\hline Ulceration & $14(63.6 \%)$ \\
\hline \multicolumn{2}{|l|}{ Histopathological assessment: } \\
\hline Thrombotic & $4(18.2 \%)$ \\
\hline Lipid rich & $7(31.8 \%)$ \\
\hline Fibrotic & $4(18.2 \%)$ \\
\hline Heterogeneous & $5(22.7 \%)$ \\
\hline Calcified & $2(9.1 \%)$ \\
\hline
\end{tabular}

*Significant coronary artery stenosis exceeding 50\% lumen reduction on coronary angiography, ${ }^{* *}$ according to NASCET criteria. presenting with moderate motoric aphasia, which diminished significantly on examination after 1 month of follow-up, the second one with mild left upper limb paresis, as well as atrial fibrillation in 1 patient after CEA with no further clinical consequences. No periprocedural deaths, MI, major IS or TIA were noted.

\section{Diffusion-weighted magnetic resonance imaging}

Acute and subacute multiple cerebral ischemic lesions were observed on DWI-MRI in all subjects before CAR (mean lesion size of $17 \pm 14.9 \mathrm{~mm}$, range: $4-58 \mathrm{~mm}$ ). Two patients suffered from periprocedural CIE. One had multiple ischemic lesions of maximum $24 \mathrm{~mm}$ in the left hemisphere with moderate motoric aphasia $(\mathrm{NIHSS}=5)$. At 4 weeks following CAR, the lesion decreased to $19 \mathrm{~mm}$. The second one had isolated ischemic lesions of maximum $69 \mathrm{~mm}$ with mild left upper limb paresis (NIHSS $=2$ ).

After CAR, DWI-MRI post-procedurally was contraindicated in 1 subject due to placement of the metal sternal clips.

In the remaining 18 uncomplicated patients, new MES were found in 9 (47.4\%) on 24-48 h DWI-MRI examination following CAR (Table III). In these subjects, the mean number of MES was $3.0 \pm 2.4$ (range: 1-9), with the mean lesion size of $4.3 \pm 2.2 \mathrm{~mm}$ (range: $3-8 \mathrm{~mm}$ ). Of those, in $2(22.2 \%)$ patients MES resolved completely, while in $6(66.7 \%)$ patients MES decreased on DWI-MRI at 1 month after CAR.

Table II. Details of CAR procedure techniques performed in presented subjects

\begin{tabular}{|c|c|}
\hline Parameter & Value \\
\hline CAR & 22 \\
\hline Carotid artery stenting & $17(77.3 \%)$ \\
\hline \multicolumn{2}{|l|}{ Neuroprotection system: } \\
\hline Distal & $9(52.9 \%)$ \\
\hline Proximal & $8(47.1 \%)$ \\
\hline \multicolumn{2}{|l|}{ Stent type: } \\
\hline C-Guard & $5(29.4 \%)$ \\
\hline RoadSaver & $5(29.4 \%)$ \\
\hline Carotid Wallstent & $4(23.5 \%)$ \\
\hline Cristallo & $2(11.8 \%)$ \\
\hline Xact & $1(5.9 \%)$ \\
\hline Direct stenting & $4(23.5 \%)$ \\
\hline Predilatation & $13(76.5 \%)$ \\
\hline CEA & $5(22.7 \%)$ \\
\hline Eversion & $5(100 \%)$ \\
\hline Shunt & $5(100 \%)$ \\
\hline
\end{tabular}

CAR - carotid artery revascularization, CEA - carotid endarterectomy. 
Thus, MES occurred in 1 out of 5 (20\%) patients after CEA, and 8 out of 14 (57\%) subjects after PTA. The mean diameter of MES after CEA was $4 \mathrm{~mm}$ and after PTA 3.9 \pm 2.3 , range: $1-8 \mathrm{~mm}$.

After 1 month, among all patients cerebral ischemic lesions persisted in $6(27.3 \%)$, resolved in $3(13.6 \%)$, decreased in 12 (54.5\%) and progressed in 1 (4.5\%) patient.

\section{Perfusion-weighted imaging magnetic resonance imaging}

Prior to CAR, perfusion in the hemisphere ipsilateral to CAS was significantly impaired, as compared to the contralateral hemisphere, as evidenced by longer TTP $(35.5 \pm 4.3$ vs. $35.0 \pm 4.5$ s, $p=0.042)$. As for MTT, the results failed to reach statistical significance $(22.4 \pm 3.5$ vs. $21.3 \pm 4.3 \mathrm{~s}, p=0.155)$.

At 1 month following CAR, PWI-MRI showed improvement of cerebral perfusion in the hemisphere supplied by the revascularized ICA, resulting in similar TTP and MTT parameters in both hemispheres $(32.5 \pm 3.8$ vs. $32.7 \pm 3.6 \mathrm{~s}$; $p=0.783$ and $17.9 \pm 3.3$ vs. $18.7 \pm 3.7 \mathrm{~s} ; p=0.055$ respec tively).

At 1 month, there was a significant improvement in cerebral PWI in all subjects, expressed as shortening of passage times for TTP (from mean $36.1 \pm 4.2$ to $32.6 \pm 3.9$; $p=0.0009$, range: -1.5 to $-11.9 \mathrm{~s}$ ) and for MTT (from mean $22.8 \pm 3.4$ to $17.8 \pm 3.4$; $p=0.0002$; range: -1.5 to $-10.8 \mathrm{~s})$, as compared to pre-procedural values. There was no statistical difference in brain perfusion improvement measured by MTT and TTP in patients with and without new, acute MES in brain DWI (Table III).

\section{Transcranial color-coded duplex Doppler}

Both 24-48 $\mathrm{h}$ after CAR and at 1 month following CAR, there was a significant increase of cerebral flow velocity at the site of the procedure as compared to the examination performed prior to revascularization: in the MCA from 75 \pm 19.7 to $117 \pm 33.8$ and to $98 \pm 22.7 \mathrm{~cm} / \mathrm{s}, p=0.001$, respectively and in the ACA from $76 \pm 25.9$ to $101 \pm 30.0$ and to $87 \pm 20.2 \mathrm{~cm} / \mathrm{s}, p=0.021$; respectively, Table IV.

The flow increase seen on TCD was not correlated with cerebral perfusion increase seen on PWI-MRI. The difference between post-procedural velocities and pre-procedural velocities in the MCA was not correlated with change in either TTP or MTT, both at 24-48 $\mathrm{h}$ and 1 month following CAR ( $p=N S)$.

\section{Functional and cognitive outcome}

Improvement of cognitive function was found in 16 (72.7\%) by MMSE and in 14 (63.6\%) subjects by MoCA at 1 month following CAR. MMSE scores were: 26.7 $\pm 2.2,27.4 \pm 2.0$ and $27.6 \pm 2.3$ before, at 24-48 $h$ and at 4 weeks following CAR, respectively, meaning that there was a significant improvement of cognitive function 4 weeks after CAR as compared to pre-procedural assessment $(p=0.019)$.

The MoCA scores were $23.4 \pm 3.3,23.9 \pm 3.1$ and 24.1 \pm 3.7 before, at $24-48 \mathrm{~h}$ and at 4 weeks following CAR respectively, with no significant difference between the respective examinations ( $p=0.136)$, which means no cognitive decline assessed by MoCA, despite the lack of statistically significant improvement. There was no correlation between the improvement of cerebral perfusion and improvement of MMSE and MoCA results.

Functional outcome, measured with NIHSS and mRS, significantly improved 4 weeks after CAR from $2.3 \pm 1.6$ to $1.4 \pm 1.2(p=0.012)$ and from $1.2 \pm 0.9$ to $0.7 \pm 0.9$ $(p=0.012)$, respectively (Table IV). In 7 patients NIHSS and in 9 patients mRS remained unchanged. New MES were not associated with cognitive function outcome or cerebral perfusion at 1 month after CAR as compared to pre-procedural values (Table III).

Table III. Comparison of functional and cognitive status, as well as cerebral perfusion at 1 month in patients with and without MES at 24-48 $\mathrm{h}$ following CAR

\begin{tabular}{lccc} 
Variable & $\begin{array}{c}\text { Subjects with acute MES } \\
\text { after CAR } \\
(n=9)\end{array}$ & $\begin{array}{c}\text { Subjects without acute MES } \\
\text { after CAR } \\
(n=10)\end{array}$ & P-value \\
\hline NIHSS change before vs. 1 month after CAR & $-0.8 \pm 0.9$ & $-1.1 \pm 1.0$ & 0.505 \\
\hline mRS change before vs. 1 month after CAR & $-0.4 \pm 0.5$ & $-0.6 \pm 0.5$ & 0.450 \\
\hline MMSE change before vs. 1 month after CAR & $1.0 \pm 1.5$ & $0.9 \pm 1.9$ & 0.902 \\
\hline MoCA change before vs. 1 month after CAR & $0.3 \pm 2.3$ & $1.5 \pm 2.8$ & 0.437 \\
\hline TTP change before vs. 1 month after CAR & $-3.51 \pm 2.63$ & $-3.72 \pm 4.21$ & 0.911 \\
\hline MTT change before vs. 1 month after CAR & $-6.35 \pm 2.51$ & $-3.42 \pm 3.11$ & 0.112
\end{tabular}

TTP - time to peak, MTT - mean transit time, MMSE - Mini-Mental State Examination, MoCA - Montreal Cognitive Assessment, NIHSS - National Institute of Health Stroke Scale, $m R S$ - modified Rankin Scale.

*One patient had no DWI-MRI after CAR due to sternal metal clips; in 2 patients PTA was complicated by minor IS with new ischemic lesions on brain MRI. Data are presented as the difference between pre-procedural and 1-month follow-up values. 
Table IV. Cognitive and functional outcome as well as cerebral perfusion in TCD and brain MRI before, 24-48 $\mathrm{h}$ after and 1 month after CAR in patients with symptomatic carotid artery stenosis

\begin{tabular}{lcccc} 
Variable & Before CAR & 24-48 h after CAR & After 4-week follow-up & $P$-value \\
\hline TCD: & & & & \\
\hline iMCA PSV [cm/s] & $75 \pm 19.7$ & $117 \pm 33.8$ & $98 \pm 22.7$ & 0.001 \\
\hline iACA PSV & $76 \pm 25.9$ & $101 \pm 30.0$ & $87 \pm 20.2$ & 0.021 \\
\hline CMCA PSV & $122 \pm 34.0$ & $119 \pm 23.8$ & $106 \pm 22.9$ & 0.026 \\
\hline CACA PSV & $125 \pm 38.9$ & $96 \pm 22.2$ & $88 \pm 23.9$ & 0.005 \\
\hline Functional status: & & & $1.4 \pm 1.2$ & 0.003 \\
\hline NIHSS & $2.3 \pm 1.6$ & $2.3 \pm 1.7$ & $0.7 \pm 0.9$ & 0.005 \\
\hline mRS & $1.2 \pm 0.9$ & $1.3 \pm 1.0$ & $27.6 \pm 2.3$ & 0.019 \\
\hline Cognitive function: & & & $24.1 \pm 3.7$ & 0.136 \\
\hline MMSE & $26.7 \pm 2.2$ & $27.4 \pm 2.0$ & & \\
\hline MoCA & $23.4 \pm 3.3$ & $23.9 \pm 3.1$ & $32.5 \pm 3.8$ & 0.0009 \\
\hline PWI: & & & $17.9 \pm 3.3$ & 0.0002
\end{tabular}

TTP - time to peak, MTT - mean transit time, MMSE - Mini-Mental State Examination, MoCA - Montreal Cognitive Assessment, NIHSS - National Institute of Health Stroke Scale, $m R S$ - modified Rankin Scale, TCD - transcranial color-coded duplex Doppler, MCA - middle cerebral artery, ACA - anterior cerebral artery.

\section{Discussion}

In this preliminary study concerning a variety of factors that may potentially impact the result of CAR in patients after first in their lifetime CIE, we observed the immediate improvement of cerebral flow in terms of the velocity increase in MCA and ACA in the circle of Willis, and cerebral perfusion, as well as a significantly improved functional outcome in all subjects.

In the light of the CIE recurrence rate as well as high 1-year mortality and disability rate, accounting for up to $50 \%, 20 \%$ and $30-40 \%$, respectively, urgent intervention for CAS seems highly justified [4, 7].

We would like to emphasize several aspects of CIE related to CAS. As evidenced by others, and also observed in the present study, the majority of symptomatic patients have carotid plaque morphology which could be called a vulnerable plaque [19]. In subjects with CIE due to CAS, echolucent plaques accounted for $71 \%$ of all plaques identified, ulcerated in $67 \%$. Several studies have already confirmed that hypoechogenic, echolucent carotid plaques increase the risk of CIE in patients with CAS and can be a viable predictor of prevalence of future CIE in those patients [26-29].

Furthermore, the collateral cerebral circulation is less prevalent in recently symptomatic patients as compared to asymptomatic with CAS and was observed in 12 (57.1\%) of 21 study participants. These findings are in line with our former study on 371 patients, in which the collateral flow within the circle of Willis as observed by TCD was present in $67 \%$ of patients with recent CIE [20]. Similarly, in the preliminary study by Ito et al., on
12 subjects, the presence of the ACoA and PCoA on dynamic magnetic resonance angiography was observed in $42 \%$ and $17 \%$ of symptomatic patients with CAS > $70 \%$, respectively [30].

Our study demonstrated that cerebral perfusion on PWI-MRI prior to CAR was significantly impaired within the hemisphere supplied by the stenotic ICA. Following CAR, we observed similar PWI parameters in both hemispheres. There are limited data concerning this aspect of CAR in the available literature. Piñero et al. observed significantly increased TTP in all patients prior to PTA in the affected hemisphere in comparison to the contralateral hemisphere $(p=0.007)$ [31]. Similar to our study, normalization of cerebral perfusion was observed for TTP 1 month after PTA in all patients [31].

What is more, cerebral perfusion improved significantly in both hemispheres, which was expressed by the shortening of TTP and MTT bilaterally. A similar outcome in regard to TTP measured briefly after CAR was noted in most, but not all, patients in the study by Gauvrit et al. [32], as well as Tavares et al. [33] and Wang et al. [34].

After CAR, there was an immediate increase in the flow velocities in the ipsilateral MCA and ACA observed in the TCD study $24-48 \mathrm{~h}$ following the procedure, which stabilized in the study after 4 weeks of follow-up. Those findings correspond well with another study on 92 patients, which found a significant PSV increase by $26 \%$ in the MCA and by $30 \%$ in the ACA measured by TCD $24 \mathrm{~h}$ after PTA as compared to examination prior to CAR [35]. These results are also in line with available studies con- 
firming the improvement of circle of Willis flow by either TCD or MRI examination [36, 37].

In our study, the increase of brain perfusion was followed by the improvement of functional outcome and cognitive functions. Concordantly, in the study by Wang et al. [34] concerning 46 patients treated with CEA, PWI improvement after CAR was correlated with the improvement in MoCA $(20.5 \pm 1.7$ vs. $22.0 \pm 1.5, p=0.001)$ with a linear correlation between TTP and MoCA $(R=-0.893$, $p<0.001)$. In our study there was a statistically significant improvement in MMSE $(26.5 \pm 2.2$ vs. $27.9 \pm 2.0$, $p=0.014)$, but not in MoCA (23.2 \pm 3.2 , vs. $24.3 \pm 3.2$, $p=0.171)$. In the only available, preliminary study on 20 patients, concerning the effect of PTA on perfusion and cognitive outcome, the improvement of brain perfusion was a strong predictor of cognitive improvement $(p=0.04)$ [38].

The inevitable consequence of CAR is MES, which are more evident after PTA as compared to CEA. It is still open for discussion whether, and to what degree, new MES impact cognitive function, functional status and brain perfusion.

In our study, there was no association between new MES in DWI-MRI and cognitive and functional status. The incidence of new MES observed in DWI-MRI 24-48 h after PTA was 7/13 (54\%), while after CEA it was $1 / 5$ (20\%), which is consistent with the available literature [13-16, $39,40]$.

However, despite the fact that $40.9 \%$ of our patients had evidence of new MES on DWI-MRI, this finding was not associated with poorer functional and cognitive outcome, and it did not have an impact on the cerebral perfusion improvement. It is worth mentioning that, in our study, MES were small in size. Furthermore, in 15\% of subjects, MES resolved after 1 month, while in the remaining 52\% they decreased significantly. Similarly, Grunwald et al. reported that the presence of DWI lesions did not affect the neuropsychological change, and PTA itself did not lead to cognitive decline [13]. Those findings suggest that although CAR is associated with the acute procedure-related MES, they do not clearly contribute to cognitive deprivation or dementia [13].

In contrast, in the study by Maggio et al. [15], the lesion incidence on DWI-MRI in patients after PTA was $24 \%$ and the incidence of new MES after PTA was associated with a significant decrease of cognitive outcome assessed by MMSE: mean MMSE reduction of -3.1 $(95 \% \mathrm{Cl}$ ranging from -5.8 to -0.5$)$ in patients with new MES after CAR, as compared to mean MMSE change of +1.1 $(95 \% \mathrm{Cl}$ from -0.3 to +2.5$)$ in the group with no new lesions [15]. Similar findings can be found in the paper by Zhou et al. [16], in which the multivariate regression analysis revealed that MES observed after CAR were associated with memory decline $(p=0.016)$ and overall cognitive deterioration in MMSE $(p=0.026)$ [16].
To summarize, our findings, as well as available data, indicate that early revascularization after CIE seems to have a beneficial effect on cerebral perfusion, functional status and cognitive function, despite the fact that the revascularization procedure carries the risk of periprocedural microembolisation.

\section{Conclusions}

This preliminary study concerning many aspects of CAR following CIE indicated immediate improvement of cerebral flow seen on TCD and brain perfusion in PWI MRI. However, in our study no correlation was observed between the improvement of brain perfusion in PWI-MRI and cerebral flow in TCD examination. We observed an improvement in cognitive function in about $2 / 3$ of patients, as well as in the functional status in the majority of our patients. Our study also demonstrated no correlation between cognitive function changes and new MES after carotid revascularization. Larger, preferably multicenter studies are necessary to elucidate the association between cognitive function, functional status and brain perfusion.

\section{Acknowledgments}

The study was supported from the Jagiellonian University research grant: K/ZDS/005730.

\section{Conflict of interest}

The authors declare no conflict of interest.

\section{References}

1. Rothwell PM, Coull AJ, Silver LE, et al. Population-based study of event-rate, incidence, case fatality, and mortality for all acute vascular events in all arterial territories (Oxford Vascular Study). Lancet 2005; 366: 1773-83.

2. Munsch F, Sagnier S, Asselineau J, et al. Stroke location is an independent predictor of cognitive outcome. Stroke 2016; 47: 66-73.

3. STROKE PREVENTION RESEARCH UNIT, Department of Clinical Neurology OU, Level 6, West Wing, John Radcliffe Hospital, Oxford, OX3 9DU U. Model for predicting the risk of ipsilateral ischaemic stroke in patients with recently symptomatic carotid bifurcation stenosis. Available from: http://www.stroke.ox.ac. uk/model/form 1.html

4. Lovett JK, Coull AJ, Rothwell PM. Early risk of recurrence by subtype of ischemic stroke in population-based incidence studies. Neurology 2004; 62: 569-73.

5. Connolly M, Bilgin-Freiert A, Ellingson B, et al. Peripheral vascular disease as remote ischemic preconditioning, for acute stroke. Clin Neurol Neurosurg 2013; 115: 2124-9.

6. Kabłak-Ziembicka A, Przewłocki T, Stępień E, et al. Relationship between carotid intima-media thickness, cytokines, atherosclerosis extent and a two-year cardiovascular risk in patients with arteriosclerosis. Kardiol Pol 2011; 69: 1024-31.

7. Tendera M, Aboyans V, Bartelink ML, et al. ESC Guidelines on the diagnosis and treatment of peripheral artery diseases: doc- 
ument covering atherosclerotic disease of extracranial carotid and vertebral, mesenteric, renal, upper and lower extremity arteries: the Task Force on the Diagnosis and Treatment of Peripheral Artery Diseases of the European Society of Cardiology (ESC). Eur Heart J 2011; 32: 2851-906.

8. Brott TG, Halperin JL, Abbara S, et al. 2011ASA/ACCF/AHA/ AANN/AANS/ACR/ASNR/CNS/SAIP/SCAI/SIR/SNIS/SVM/SVS Guideline on the management of patients with extracranial carotid and vertebral artery disease. J Am Coll Cardiol 2011; 57: e16-94.

9. Cheng Y, Wang YJ, Yan JC, et al. Effects of carotid artery stenting on cognitive function in patients with mild cognitive impairment and carotid stenosis. Exp Ther Med 2013; 5: 1019-24.

10. Kablak-Ziembicka A, Przewłocki T. Carotid artery stenting drawbacks: microembolic ischemic cerebral lesions - do they matter? J Endovasc Ther 2011; 18: 527-30.

11. Plessers M, Van Herzeele I, Vermassen F, et al. Neurocognitive functioning after carotid revascularization: a systematic review. Cerebrovasc Dis 2014; 4: 132-48.

12. Rothwell PM, Warlow CP. Prediction of benefit from carotid endarterectomy in individual patients: a risk-modelling study. European Carotid Surgery Trialists' Collaborative Group. Lancet 1999; 353: 2105-10.

13. Grunwald IQ, Papanagiotou P, Reith W, et al. Influence of carotid artery stenting on cognitive function. Neuroradiology 2010; 52: 61-6.

14. Gossetti B, Gattuso R, Irace L, et al. Embolism to the brain during carotid stenting and surgery. Acta Chir Belg 2007; 107: 151-4.

15. Maggio P, Altamura C, Landi D, et al. Diffusion-weighted lesions after carotid artery stenting are associated with cognitive impairment. J Neurol Sci 2013; 328: 58-63.

16. Zhou W, Hitchner E, Gillis K, et al. Prospective neurocognitive evaluation of patients undergoing carotid interventions. J Vasc Surg 2012; 56: 1571-8.

17. Pieniążek P, Tekieli L, Musiałek P, et al. Carotid artery stenting according to the tailored-CAS algorithm is associated with a low complication rate at 30 days: data from the TARGET-CAS study. Kardiol Pol 2012; 70: 378-86.

18. Carpenter JP, Lexa FJ, Davis JT. Determination of duplex Doppler ultrasound criteria appropriate to the North American Symptomatic Carotid Endarterectomy Trial. Stroke 1996; 27: 695-9.

19. Gray-Weale AC, Graham JC, Burnett JR, et al. Carotid artery atheroma: comparison of preoperative B-mode ultrasound appearance with carotid endarterectomy specimen pathology. J Cardiovasc Surg 1988; 29: 676-81.

20. Badacz R, Przewłocki T, Karch I, et al. Low prevalence of collateral cerebral circulation in the circle of Willis in patients with severe carotid artery stenosis and recent ischemic stroke. Postep Kardiol Inter 2015; 11: 312-7.

21. Nasreddine ZS, Phillips NA, Bedirian V, et al. The Montreal Cognitive Assessment, MoCA: a brief screening tool for mild cognitive impairment. J Am Geriatr Soc 2005; 53: 695-9.

22. Vogel SJ, Banks SJ, Cummings JL, et al. Concordance of the Montreal cognitive assessment with standard neuropsychological measures. Alzheimer's Dement 2015; 1: 289-94.

23. Philipps V, Amieva H, Andrieu S, et al. Normalized mini-mental state examination for assessing cognitive change in population-based brain aging studies. Neuroepidemiology 2014; 43: $15-25$.
24. Goldstein LB, Samsa GP. Reliability of the National Institutes of Health Stroke Scale. Extension to non-neurologists in the context of a clinical trial. Stroke 1997; 28: 307-10.

25. Nunn A, Bath PM, Gray LJ. Analysis of the modified Rankin scale in randomised controlled trials of acute ischaemic stroke: a systematic review. Stroke Res Treat 2016; 2016: 9482876.

26. Hendrikse J, Hartkamp MJ, Hillen B, et al. Collateral ability of the circle of willis in patients with unilateral internal carotid artery occlusion: border zone infarcts and clinical symptoms. Stroke 2001; 32: 2768-73.

27. Biasi GM, Froio A, Diethrich EB, et al. Carotid plaque echolucency increases the risk of stroke in carotid stenting: the Imaging in Carotid Angioplasty and Risk of Stroke (ICAROS) study. Circulation 2004; 110: 756-62.

28. Grønholdt ML, Nordestgaard BG, Schroeder TV, et al. Ultrasonic echolucent carotid plaques predict future strokes. Circulation 2001; 104: 68-73.

29. Musialek P, Tracz W, Tekieli L, et al. Multimarker approach in discriminating patients with symptomatic and asymptomatic atherosclerotic carotid artery stenosis. J Clin Neurol 2013; 9: 165-75.

30. Ito K, Sasaki M, Kobayashi M, et al. Noninvasive evaluation of collateral blood flow through circle of willis in cervical carotid stenosis using selective magnetic resonance angiography. J Stroke Cerebrovasc Dis 2014; 23: 1019-23.

31. Piñero P, González A, Moniche F, et al. Progressive changes in cerebral perfusion after carotid stenting: a dynamic susceptibility contrast perfusion weighted imaging study. J Neurointerv Surg 2014; 6: 527-32.

32. Gauvrit JY, Delmaire C, Henon $H$, et al. Diffusion/perfusionweighted magnetic resonance imaging after carotid angioplasty and stenting. J Neurol 2004; 251: 1060-7.

33. Tavares A, Caldas JG, Castro CC, et al. Changes in perfusionweighted magnetic resonance imaging after carotid angioplasty with stent. Interv Neuroradiol 2010; 16: 161-9.

34. Wang Q, Zhou M, Zhou Y, et al. Effects of carotid endarterectomy on cerebral reperfusion and cognitive function in patients with high grade carotid stenosis: a perfusion weighted magnetic resonance imaging study. Eur J Vasc Endovasc Surg 2015; 50: 5-12.

35. Kablak-Ziembicka A, Przewlocki T, Pieniazek P, et al. Assessment of flow changes in the circle of Willis after stenting for severe internal carotid artery stenosis. J Endovasc Ther 2006; 13: 205-13.

36. Youn SW, Kim HK, Do YR, et al. Haemodynamic alterations in cerebral blood vessels after carotid artery revascularisation: quantitative analysis using 2D phase-contrast MRI. Eur Radiol 2013; 23: 2880-90.

37. Shakur SF, Amin-Hanjani S, Bednarski C, et al. Intracranial blood flow changes after extracranial carotid artery stenting. Neurosurgery 2015; 76: 330-6.

38. Moftakhar R, Turk AS, Niemann DB, et al. Effects of carotid or vertebrobasilar stent placement on cerebral perfusion and cognition. AJNR Am J Neuroradiol 2005; 26: 1772-80.

39. Schnaudigel S, Gröschel K, Pilgram SM, et al. New brain lesions after carotid stenting versus carotid endarterectomy: a systematic review of the literature. Stroke 2008; 39: 1911-9.

40. Sahin N, Solak A, Genc B, et al. Brain diffusion changes in unilateral carotid artery stenosis with non-shunt endarterectomy: correlation with white matter lesions. Clin Neurol Neurosurg 2015; 133: 24-9. 